\title{
Demandas por Malpraxis en Distintas Etapas del Tratamiento en Implantología Oral: Una Revisión
}

\author{
Malpractice Claims at Different Stages of Treatment In Oral Implantology: A Review
}

\author{
Matías Arroyo-Navarrete ${ }^{1} \&$ Gabriel M. Fonseca ${ }^{2}$
}

\begin{abstract}
ARROYO-NAVARRETE, M. \& FONSECA, G. M. Demandas por malpraxis en distintas etapas del tratamiento en implantología oral: Una revisión. Int. J. Odontostomat., 15(2):434-442, 2021.

RESUMEN: Las demandas por malpraxis en odontología se han incrementado en los últimos años, siendo la implantología una de las especialidades más litigadas. Estas demandas en su mayoría se han caracterizado por tener un carácter multifactorial, con errores reportados en cualquiera de sus fases diagnósticas, terapéuticas o de mantenimiento. El propósito de esta revisión fue establecer la etapa del tratamiento implantológico en la que más se realizaron demandas por malpraxis, estableciendo una categorización de los errores detectados y de los daños asociados a cada una de las fases que incluye el tratamiento de rehabilitación mediante implantes dentales. Se realizó una revisión con búsqueda sistemática de los últimos 10 años en las bases Pubmed, Scopus, Web of Science, SciELO, complementada con una búsqueda manual en revistas especializadas y en Google Scholar de artículos a partir de términos clave en idiomas español inglés y portugués. Se identificaron solo 3 artículos que cumplieron los criterios de selección, lo que afirma el concepto de escasa atención hacia esta eventualidad. Las demandas identificadas en esos reportes fueron analizadas sobre cuatro tipos de riesgo en implantología según la etapa del tratamiento en la que aparecen. La etapa quirúrgica fue identificada como la de mayor potencial de riesgo de originar eventos adversos y demandas asociadas. Se sugiere profundizar en los aspectos medicolegales propios y genéricos de la especialidad, como así también en el desarrollo de estrategias que prevengan sus eventos adversos y la judicialización asociada.
\end{abstract}

PALABRAS CLAVE: Implantología oral, malpraxis, prevención, riesgo sanitario.

\section{INTRODUCCIÓN}

A lo largo de los años, los tratamientos de atención médica se han asociado a una serie de eventos adversos, riesgos y errores, siendo estos hechos de gran importancia para el clínico en la práctica diaria. Para el profesional el objetivo primordial será lograr el mejor resultado posible al finalizar el tratamiento, por lo que en caso de no darse el resultado esperado $u$ otra complicación una vez concluido este, el paciente podría optar a realizar una demanda judicial para compensar dicha situación.

Esto último, ha ido tomando aún más importancia en los últimos años, debido al énfasis que se le ha dado actualmente al respeto por la dignidad humana y la toma de decisiones libres y consentidas por parte del paciente, cuyas ideas se originan en el año 1981 en la Declaración de Lisboa realizada por la Asociación Médica Mundial. Consecuentemente, en el año 2002 la World Health Organization (WHO) instó a sus países miembros a mejorar la seguridad del paciente y los sistemas de monitoreo, a través de una resolución que además pretendía establecer redes de investigación y promoción en materias de seguridad, esperando dar estrategias preventivas apropiadas ante el surgimiento de estas (World Health Organization, 2002). Por su parte, en el año 2012 en Chile se promulgó la Ley №20.584 que regula los Derechos y Deberes del paciente en relación a acciones vinculadas con atención en salud, la cual provocó un cambio radical en la relación médico-paciente al establecer

\footnotetext{
${ }^{1}$ Carrera de Odontología, Facultad de Odontología, Universidad de La Frontera, Temuco, Chile.

${ }^{2}$ Centro de Investigación en Odontología Legal y Forense (CIO), Facultad de Odontología, Universidad de La Frontera, Temuco, Chile.
} 
una relación orientada hacia la beneficencia, respetando la autonomía y toma de decisiones del usuario (Ministerio de Salud, 2012) siendo esto reflejado en el reconocimiento al consentimiento informado o en establecimiento de Comités de Ética.

Pese a ello, se ha visto que las demandas por malpraxis en odontología han incrementado en los últimos años, siendo una de las áreas de la salud que reciben mayor cantidad de litigios por ello (Knaak \& Parzeller, 2014). Estas demandas se han caracterizado por tener un carácter multifactorial, incluyendo causas como negligencia, errores técnicos, el atropello del respeto y la dignidad del usuario, la falta de documentación o la mala comunicación entre paciente-operador, siendo esta última una de las principales causas de reclamos en relación a las prácticas odontológicas (Triana Estrada, 2006). La especialidad de implantología no es la excepción la cual, considerando adicionalmente los altos costos en el tratamiento, es una de las prácticas odontológicas que conlleva más riesgos de causar daños al paciente por complicaciones de todo tipo (Misch \& Wang, 2008). Respecto a las causas más frecuentes de falla tardía del tratamiento de implantes, se pueden reconocer aquellas relacionadas con la sobrecarga oclusal o estrés excesivo, con periimplantitis y mala higiene oral, como así también aquellas relacionadas con el diseño, construcción y ajuste incorrecto de la prótesis (Tolstunov, 2006), pudiendo darse cualquiera de estas complicaciones en cualquier etapa del tratamiento.

En Chile, se ha reportado que los tratamientos de rehabilitación con implantes dentales son de los más reclamados por malpraxis (Portela León, 2013; Moscoso Matus \& Smok Vásquez, 2015), donde la mayoría de las reclamaciones van asociadas a pretensiones económicas o exigencias de devolución del dinero, lo que supone un quiebre en la relación entre el paciente y profesional dado por un tratamiento mal efectuado o por poca comunicación entre ambos (Portela León). Tomando esto en consideración, el Decreto Exento $N^{\circ} 31$ del Ministerio de Salud de Chile que modifica su Norma Técnica $\mathrm{N}^{\circ} 145$, establece que el especialista de Implantología debe poseer competencias y conocimientos mínimos para la certificación de la especialidad en cuestión, además de contar con cierta experiencia y contenidos teórico-prácticos mínimos para la ejecución de la disciplina, por lo que es imprescindible que el implantólogo tenga un conocimiento actualizado de las opciones y alternativas terapéuticas disponibles, realizando las correctas indicaciones de exámenes complementarios y derivaciones correspondientes para un resultado final exitoso (Ministerio de Salud, 2013). Asimis- mo, se aconseja establecer el uso constante del consentimiento informado, señalando al usuario las implicancias y riesgos de la acción a realizar para lograr mantener una buena comunicación paciente-operador desde un principio, evitando complicaciones legales a futuro (Givol et al., 2002; Misch \& Wang; Rees, 2013)

Se presenta una revisión con búsqueda sistemática para establecer la etapa del tratamiento implantológico en la que más se realizan demandas por malpraxis, estableciendo una categorización de los errores terapéuticos y daños asociados a cada una de las fases que incluye el tratamiento de implantes dentales. Se discuten las causas asociadas en relación a estas fases, los riesgos inherentes y las estrategias para prevenirlas.

\section{MATERIAL Y MÉTODO}

Se realizó una búsqueda en bases de datos Pubmed, Scopus, Web of Science, Scielo, como así también una búsqueda manual en revistas pertenecientes al JCR y en la plataforma Google Scholar para una búsqueda más profunda en la literatura. Se incluyeron artículos originales, excluyendo revisiones sistemáticas, tesis, reportes de casos y libros, o aquellos artículos que no especificaran las causas directas asociadas a las demandas estudiadas. Se utilizaron como límites los estudios realizados en humanos durante los últimos 10 años, considerando artículos en idiomas español, inglés y portugués. La búsqueda se realizó entre los días 22 y 23 de mayo de 2020 y su estrategia incluyó el empleo de los términos ((implant*) and ("dental" or "dentistry" or "teeth" or "tooth")) and ("malpractice" or "claims" or "law" or "litigation" or "legislation" or "lawsuit" or "iatrogenesis", adaptándola según la base pesquisada. La búsqueda se complementó mediante la identificación de los términos clave "Claims", "Malpractice", "Litigation", "Lawsuit", "Legislation", "latrogenesis" en las revistas especializadas pertenecientes al Journal Citation Report (JCR): Clinical Implant Dentistry And Related Research, Clinical Oral Implants Research, European Journal of Oral Implantology, Implant Dentistry, Implantologie, Journal of long-term effects of Medical Implants, Journal of Oral Implantology, Journal of Periodontal and Implant Science, Journal of Prosthodontics-Implant Esthetic and Reconstructive Dentistry; a Scopus: International Journal of Oral and Maxillofacial Implants, European Journal of Implant and Refractive Surgery; y a SciELO: Avances en Periodoncia e Implantología 
Oral, Revista Clínica de Periodoncia, Implantología y Rehabilitación Oral. Tomando en cuenta artículos citados en esa primera fase, se realizó una pesquisa manual en Google Scholar desarrollando la sistematización de la búsqueda.

Una vez identificados los artículos, las causas de las demandas reportadas fueron asignadas a una o varias etapas terapéuticas siguiendo los criterios establecidos por Misch \& Wang y por Resnik (2020), clasificándolos por consenso de los autores según 4 tipos de riesgos en implantología: Riesgos asociados a Fase Quirúrgica, Riesgos asociados a Fase Protésica, Riesgos asociados a Fase Mantención y Riesgos Transversales a cada Fase. Estos cuatro tipos fueron descritos estableciendo el comienzo y término de cada una de ellos, los riesgos asociados y las especialidades del equipo multidisciplinario implantológico que participa en cada una de las etapas.

\section{RESULTADOS}

Se identificó un total de 1117 artículos, de los cuales 3 fueron finalmente incluidos por ajustarse a los criterios de búsqueda (Fig. 1). Los reportes provinieron de España, Italia y Finlandia con 35, 518 y 268 causales demandados respectivamente por malpraxis en implantología oral $(n=821)$. En Italia particularmente, los 518 causales correspondieron a 411 implantes colocados. Se observó gran heterogeneidad en la estructura y descriptiva de esos reportes (Tabla I). La asignación a la etapa terapéutica de las causas demandadas así como la/s especialidad/es asociadas, son expuesta en la Tabla II. No fueron considerados para esta categorización los tiempos Preoperatorio, Intraoperatorio y Postoperatorio debido a la poca especificación de las causales relacionadas con las demandas en cuestión.

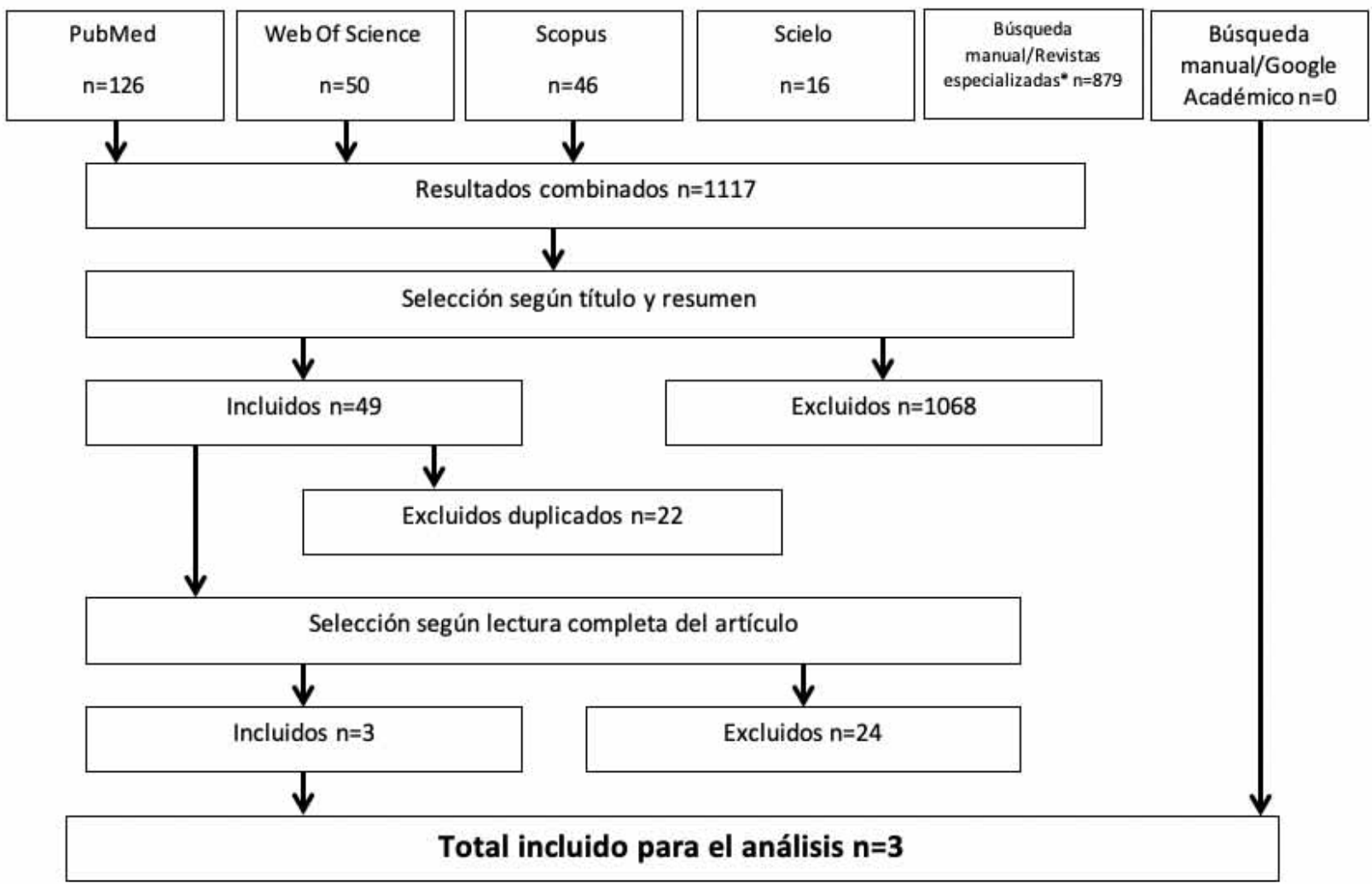

Fig. 1. Flujograma de Selección de Artículos *Revistas incluidas pertenecientes al JCR: Clinical Implant Dentistry And Related Research, Clinical Oral Implants Research, European Journal of Oral Implantology, Implant Dentistry, Implantologie, Journal of long-term effects of Medical Implants, Journal of Oral Implantology, Journal of Periodontal and Implant Science, Journal of Prosthodontics-Implant Esthetic and Reconstructive Dentistry; a Scopus: International Journal of Oral and Maxillofacial Implants, European Journal of Implant and Refractive Surgery; y a SciELO: Avances en Periodoncia e Implantología Oral, Revista Clínica de Periodoncia, Implantología y Rehabilitación Oral. 
Tabla I. Análisis de artículos seleccionados.

\begin{tabular}{|c|c|c|c|c|c|c|}
\hline Autores & $\begin{array}{l}\text { País de } \\
\text { muestreo }\end{array}$ & $n$ & $\begin{array}{c}\text { Fase } \\
\text { Quirúrgica }\end{array}$ & $\begin{array}{c}\text { Fase } \\
\text { Protésica }\end{array}$ & $\begin{array}{c}\text { Fase } \\
\text { Mantención }\end{array}$ & Transversales \\
\hline $\begin{array}{c}\text { Marinescu Gava et al. } \\
\text { (2018) }\end{array}$ & Finlandia & 268 implantes & $\begin{array}{c}268 \\
(100,00 \%)\end{array}$ & $\begin{array}{c}0 \\
(0,0 \%)\end{array}$ & $\begin{array}{c}0 \\
(0,0 \%)\end{array}$ & $\begin{array}{c}0 \\
(0,00 \%)\end{array}$ \\
\hline Pinchi et al. (2014) & Italia & $\begin{array}{l}411 \text { implantes ( } 518 \\
\text { causales) }\end{array}$ & $\begin{array}{c}273 \\
(52,70 \%)\end{array}$ & $\begin{array}{c}33 \\
(6,37 \%)\end{array}$ & $\begin{array}{c}37 \\
(7,14 \%)\end{array}$ & $\begin{array}{c}175 \\
(33,78 \%)\end{array}$ \\
\hline Perea-Pérez et al. (2011) & España & $\begin{array}{c}35 \text { casos } \\
\text { Implantología }\end{array}$ & $\begin{array}{c}26,66(76,17 \\
\%)\end{array}$ & $\begin{array}{c}3,33 \\
(9,51 \%)\end{array}$ & $\begin{array}{c}0 \\
(0,00 \%)\end{array}$ & $\begin{array}{c}4,99 \\
(14,26 \%)\end{array}$ \\
\hline
\end{tabular}

Tabla II. Asignación a la etapa terapéutica de las causas demandadas.

\begin{tabular}{|c|c|c|c|}
\hline Etapa & Descripción & Situaciones incluidas & $\begin{array}{c}\text { Especialidades } \\
\text { asociadas }\end{array}$ \\
\hline $\begin{array}{c}\text { Riesgos } \\
\text { asociados a } \\
\text { Fase } \\
\text { Quirúrgic a }\end{array}$ & $\begin{array}{l}\text { Son aquellos riesgos que aparecen en I a } \\
\text { realización del procedimiento quirúrgico para } \\
\text { la inserción del implante o fijación, pudiendo } \\
\text { darse complicaciones relacionadas con fallas } \\
\text { en previa planificación de la cirugía de } \\
\text { implantes o con compromisos a estructuras } \\
\text { anatómicas circundantes, como estructuras } \\
\text { vasculares, nerviosas, senos paranasales, } \\
\text { entre otros. Dependiendo de cada caso, esta } \\
\text { colocación se puede realizar en una o dos } \\
\text { etapas o actos quirúrgicos. Posterior a la fase } \\
\text { quirúrgica, el paciente deberá esperar entre 2- } \\
3 \text { meses para una correcta oseointegración } \\
\text { del implante y así pasar a la Fase Protésica. }\end{array}$ & $\begin{array}{ll}- & \text { Mala posición del implante } \\
- & \text { Compromiso de estructuras } \\
\text { circundantes } \\
- & \text { Daño a estructuras anatómic as } \\
- & \text { Ingestión/Deglución } \\
- & \text { Fallo en planifica ción previa a } \\
\text { cirugía } & \\
-\quad & \text { Falta de hueso disponible para } \\
\text { correcta oseointegración del } \\
\text { implante } \\
-\quad \text { Complicaciones hemorrágicas } \\
-\quad \text { Otros (Muerte) }\end{array}$ & $\begin{array}{l}\text { Cirugía Oral y } \\
\text { Maxilofacial }\end{array}$ \\
\hline $\begin{array}{c}\text { Riesgos } \\
\text { asociados a } \\
\text { Fase } \\
\text { Protésica }\end{array}$ & $\begin{array}{l}\text { Refiere aquellos riesgos que ocurren durante } \\
\text { la etapa de confección de la prótesis, la que } \\
\text { se atornilla a la base del implante que actúa } \\
\text { como raíz artificial, donde los errores más } \\
\text { comunes refieren a cargas biomecánicas } \\
\text { inapropiadas sobre el implante, pudiendo } \\
\text { provocar daños en la ATM por dichas cargas, } \\
\text { aflojamientos of racturas del tornillo de } \\
\text { fijación, traumas a e structuras óseas por } \\
\text { fuerzas iatrogénicas, entre otros. Se pueden } \\
\text { dar implantes unitarios, unidas a una sola } \\
\text { pieza cerámica, como así también podemos } \\
\text { colocar prótesis completas totalmente } \\
\text { atornilladas sobre 6-8 implantes o incluso } \\
\text { prótesis sujetas a dispositivos de anclajes } \\
\text { atornillados sobre } 3-4 \text { implantes. }\end{array}$ & $\begin{array}{ll}\text { - } & \text { Carga mecánic a inapropiada } \\
\text { - } & \text { Incorrecta oseointegración para } \\
\text { colocación de prótesis. } \\
\text { - } \quad \text { Fracturas del tornillo de soporte } \\
\text { - } \quad \text { Fractura o pérdida del implante } \\
\text { o prótesis } \\
\text { - } \quad \text { Trauma óseo } \\
\text { - Daño a ATM por cambios en } \\
\quad \text { carga mecánica } \\
\text { - Estética de prótesis inadecuada, } \\
\text { o insatisfactoria para el paciente. }\end{array}$ & $\begin{array}{l}\text { Rehabilitación } \\
\text { Oral }\end{array}$ \\
\hline $\begin{array}{c}\text { Riesgos } \\
\text { asociados a } \\
\text { Fase } \\
\text { Mantención }\end{array}$ & $\begin{array}{l}\text { Las revisiones de mantenimiento van dirigidas } \\
\text { a controlar la evolución del tratamiento } \\
\text { detectando precozmente cualquier situación } \\
\text { desfavorable que pudiera producirse posterior } \\
\text { a la finalización de este. Los problemas } \\
\text { relacionados a la mantención se asocian a } \\
\text { enfermedades periimplantarias o pérdida de la } \\
\text { reconstrucción protésica/implante previamente } \\
\text { colocados por diversos factores, pudiendo } \\
\text { darse incluso por factores propios del } \\
\text { paciente. }\end{array}$ & $\begin{array}{ll}- & \text { Periimplantitis } \\
- & \text { Mucositis Perimplantaria } \\
- & \text { Alergia o Hipersensibilidad a } \\
\text { Titanio } \\
\text { - } \\
\text { Resultado final insatisfactorio } \\
\text { para el paciente en cuanto a } \\
\text { comodidad. }\end{array}$ & Periodoncia \\
\hline $\begin{array}{l}\text { Riesgos } \\
\text { Transv ersale } \\
\text { s a cada } \\
\text { Fase } \\
\text { Terapéutica }\end{array}$ & $\begin{array}{l}\text { Son aquellos errores que tienen lugar en dos } \\
\text { o más fases terapéuticas, en donde el } \\
\text { paciente se relaciona a lo largo del } \\
\text { tratamiento con diversas áreas de } \\
\text { especialidad odontológica que integran el } \\
\text { equipo multidisciplinario. }\end{array}$ & $\begin{array}{ll}\text { _ } & \text { Falta de comunicación paciente- } \\
\text { operador } \\
\text { _ } \quad \text { Falta de documentación o } \\
\text { documentación incompleta } \\
\text { _ } \quad \begin{array}{l}\text { Necesidad de repetición de } \\
\text { tratamiento }\end{array} \\
\text { _ } \quad \text { Pérdida iatrogénica de dientes }\end{array}$ & $\begin{array}{ll}- & \text { Cirugía Oral y } \\
& \text { Maxilofacial } \\
-\quad & \text { Rehabilitación } \\
& \text { Oral } \\
-\quad & \text { Periodoncia }\end{array}$ \\
\hline
\end{tabular}


Respecto a la fase del tratamiento implantológico que más demandas recibió por malpraxis, se observó predominio de la Fase quirúrgica, la cual representó al menos el $50 \%$ de las causales totales en cada uno de los artículos seleccionados. Asimismo, la segunda categorización con más demandas recibidas fue la Transversal a cada una de las fases del tratamiento, ocupando el segundo lugar en dos de los tres estudios analizados. Las fases con menor cantidad de demandas por malpraxis fueron la Fase Protésica (2 de los 3 artículos analizados), como así también la Fase de Mantención, esta última con el mínimo porcentaje en cada uno de los estudios (Tabla I).

Considerando el $n$ total del análisis $(n=821)$ se observó que la distribución global de las demandas relacionadas a errores dados de manera particular a cada una de las tres fases terapéuticas tradicionales (quirúrgica, protésica y mantención) correspondió a un $78,07 \%$, mientras que aquellos errores dados en una o más etapas terapéuticas, es decir a nivel transversal, representaron un $21,92 \%$ del total analizado. De igual modo, considerando el número de casos según la distribución de los errores dados de manera específica en cada una de las fases tradicionales $(n=641)$, se observó que la Fase Quirúrgica correspondió a un $88,56 \%$, mientras que las Fases Protésica y de Mantención correspondieron a un 5,67 \% y un 5,77 \% respectivamente (Fig. 2).

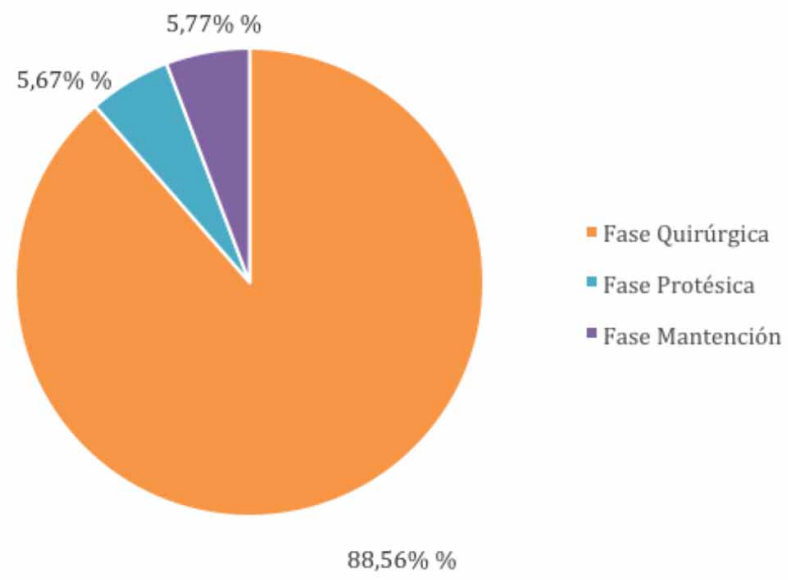

Fig. 2. Distribución específica de las demandas según etapas terapéuticas tradicionales en la que se reportó el evento.

\section{DISCUSIÓN}

El estudio de las demandas realizadas por malpraxis en odontología tiene como principal finalidad el establecer sus causas más frecuentes durante el tra- tamiento, sirviendo ésta como referencia o guía para la identificación de estos eventos adversos y para la consecuente toma de medidas preventivas. Indudablemente, y en consonancia con los estándares actuales para prevención de riesgo sanitario, los pacientes también se verían beneficiados con un profesional que toma conocimiento de las causas más frecuentes de producción de estos eventos, y adopta las precauciones y consideraciones antes, durante y después del tratamiento para así garantizar la mayor protección al paciente (Rivera-Mendoza et al., 2017).

Cabe mencionar que la literatura científica disponible sobre análisis de causas de demandas por malpraxis, carece de datos específicos, de homogeneidad de información y de cantidad de estudios comparables para el análisis. Si bien existen artículos que abordan a un nivel más general las especialidades odontológicas que reciben mayor cantidad de demandas, el número de estudios no es amplio. Asimismo, y según nuestro conocimiento, existe una escasez mayor de estudios actualizados que especifiquen las causas asociadas a las demandas realizadas, siendo los del área de implantología prácticamente inexistentes. Esto es aún más notorio en Chile, lo que impide reflejar con exactitud la realidad nacional en este estudio.

Esta escasez de estudios locales sobre demandas en odontología, es una señal de alarma debido a que los litigios por malpraxis han pasado a ser parte de la cotidianeidad de la profesión o inclusive del área de la implantología según lo demostrado en algunos estudios realizados en Portugal (Corte-Real et al., 2020), España (Bordonaba-Leiva et al., 2019) e Irán (Kiani \& Sheikhazadi, 2009). En contraste con la ausencia de reportes locales, la realidad es bastante agresiva para el odontólogo chileno: en 2019, un cirujano dentista especialista en implantología fue condenado a indemnizar a un paciente por lesiones, por un monto superior a los 100 mil dólares (Villarroel, 2019). La demanda se originó en un déficit en la planificación previa del tratamiento en cuanto al diámetro y profundidad adecuados para la inserción quirúrgica del implante, siendo esta una señal clara de que se debe profundizar en este tipo de estudios con la finalidad de ser una medida preventiva ante estos eventos adversos en el ejercicio de la especialidad en Chile.

Este caso en particular fue el gatillo respecto a la búsqueda de nuestro estudio: previendo la posibilidad de que la mayoría de las demandas por malpraxis se producen por errores generados en la Fase Quirúrgica, nuestra revisión confirmó precisamente esa fase como 
la más riesgosa y proclive a demandas (88,56 \%). Esto puede verse influenciado por factores como son la falta de experiencia del operador o el carácter invasivo que caracteriza a dicha etapa, lo que ya ha sido sugerido en la literatura (Marinescu Gava et al., 2018; Perea-Pérez et al., 2011; Pinchi et al., 2014). Los errores asociados a esta fase refieren al compromiso de estructuras circundantes (mayormente lesiones en nervios alveolar inferior y lingual) o errores en la posición o angulación del implante, todos ya descritos como eventos adversos quirúrgicos habituales (Camargo \& Van Sickels, 2015; Misch \& Wang; Resnik).

Sin embargo, en la actualidad existen diversas herramientas que podrían reducir significativamente algunas de estas complicaciones; tal es el caso de la cirugía guiada de implantes, el uso de tomografía computarizada cone beam (CBCT) o una planificación digitalizada del tratamiento (DeVico et al., 2016; Van Assche et al., 2012). Pese a ello, la aparición de estos eventos adversos específicamente en la Fase Quirúrgica es impredecible, lo que condiciona absolutamente la efectividad de cualquier medida preventiva (Camargo \& Van Sickels). La aparición de estos eventos y de sus resultados negativos durante el tratamiento tiene un impacto negativo en la vida diaria del paciente, como así también gran relevancia en cuanto a la tasa de sobrevida del implante (Chee \& Jivraj, 2007). En particular las reportadas secuelas por daños a estructuras nerviosas, más allá del fracaso implantológico, devienen en graves afecciones a la autoestima del paciente, así como a sus relaciones interpersonales y laborales. Los déficits neurológicos asociados conllevan alteración de desempeños comunes en el quehacer diario de la persona, como lo son la masticación, fonación, deglución, entre otras actividades relacionadas que afectarían el normal funcionamiento del sistema estomatognático (Pippi et al., 2018). El clínico debería tener los conocimientos apropiados en cuanto a las causas que provocan este déficit, su prevención y los tratamientos disponibles para ello, realizando asimismo un diagnóstico temprano y certero del compromiso neurológico para su oportuna derivación en caso que fuera necesario. Adicionalmente, la posible aparición de este evento adverso debe ser consignada en el consentimiento informado como así también los potenciales riesgos y complicaciones que podrían surgir en relación a estas estructuras vitales (Misch \& Resnik, 2010).

Dado que la posición y angulación intraóseas del implante favorecen el éxito o fracaso del tratamiento implantológico, fallas en ella pueden someter el dispositivo a riesgo de fractura o incluso a problemas en la oclusión de la futura prótesis. Esto provoca tensiones adicionales, mordidas cruzadas, inestabilidad, entre otras complicaciones que denotarían la relevancia de la orientación espacial del implante (Kourtis et al., 2004; Misch \& Wang). Claramente la fase quirúrgica se torna crítica y de necesario foco para la prevención de eventos adversos; sin embargo, estas fases no pueden ser analizadas de manera aislada, sino en un conjunto donde fases iniciales (diagnóstica, quirúrgica, etc.) pueden tener evidentes repercusiones futuras (fase rehabilitadora, fase de mantención). Tatakis et al. (2019) afirman que pueden ocurrir eventos adversos sustanciales en cada uno de los pasos individuales y pueden acumularse, impactando significativamente en el éxito o fracaso del tratamiento implantológico. Las estrategias generales para reducir o eliminar estos riesgos deberían incluir: el conocimiento de los posibles riesgos, de los sistemas y herramientos utilizados, de la verificación permanente tanto de los procedimientos diagnósticos y quirúrgicos y de una formación y experiencia quirúgica apropiadas.

Este estudio pone relevancia en que gran parte de las demandas surgen por eventos adversos producidos en dos o más etapas del tratamiento, transversalmente, donde destacan la documentación incompleta, la falta total de esta, o la falta de comunicación entre paciente y operador, siendo estas dos variables interrelacionables entre sí. Esto denota la gran relevancia de comunicar al paciente la mayor cantidad de posibilidades que eventualmente podrían afectar el normal curso de los procedimientos en cada una de las etapas, informando al paciente los porcentajes de riesgos previsibles, antes y después del acto quirúrgico (Pippi et al.). El Consentimiento Informado no es una nota estática o solo un requerimiento técnico para poder dar inicio al tratamiento; es un documento vivo y dinámico en el cual cada una de las premisas diagnósticas y terapéuticas debe ser informada apropiadamente y consensuada con el paciente, lo que incluye además a cambios en la dirección del tratamiento, eventos adversos y sus respuestas o las nuevas previsiones que deban realizarse por tal efecto (Fonseca et al., 2020). El consentimiento informado optimiza la comunicación con el paciente desde el principio; por lo mismo, es importante que considere entre los posibles eventos adversos del tratamiento implantológico, los fallos iatrogénicos, mecánicos y biológicos (Esposito et al., 1998), estos últimos relevantes en este caso por el hecho de ser condicionantes propias del paciente. La omisión de estos datos o el no conocimiento de estas condiciones por parte del paciente u operador podrían ser decisivas en el resultado final del tratamiento. Este 
punto podría ser considerado el más crítico en cuanto a la causa de las demandas, ya que en caso de que el clínico no informe las implicancias del tratamiento o que no esté en conocimiento de la condición de su paciente por omisión de datos, el usuario podría demandar al operador por no tomar en cuenta su decisión, ni comunicarle las posibles complicaciones que podrían surgir durante el tratamiento, las que deberían estar explícitas en el consentimiento informado previo a cada uno de los procedimientos a realizar.

La baja frecuencia de demandas por malpraxis relacionada con la Fase Protésica, se puede asociar a la poca probabilidad de aparición de eventos adversos, al ser menos invasiva en comparación a la fase previa a esta (fase quirúrgica). Asimismo, se puede suponer que la mayoría de los pacientes que pasan a esta etapa tienen como requisito el éxito previo en la oseointegración del implante, la que es iniciada en la fase quirúrgica con la finalidad de lograr una estabilidad óptima para el implante que soporte la carga mecánica de la reconstrucción protésica (Resnik). Las fallas que pueden ocurrir entre la conexión del pilar y la prótesis son causadas comúnmente por cargas desfavorables (Isidor, 1998); asimismo, en implantología debe primar el concepto de que toda colocación de implantes debe ser guiada por la prótesis, y no el implante guiar la prótesis (Collins Calcaño et al., 2014). Debemos destacar sin embargo, la baja frecuencia de demandas en la Fase Protésica arrojada en este estudio no coincide con la cantidad de demandas en el área de rehabilitación oral o prostodoncia en estudios realizados en Italia, Irán y EE.UU. (Hapcook Sr., 2006; Hashemipour et al., 2013; Kiani \& Sheikhazadi; Manca et al., 2018), los que reportan que las especialidades más demandadas por malpraxis son las relacionadas con dicha fase terapéutica. Para esos autores, los tópicos de la rehabilitación que destacan como causales son la falta de habilidad del operador, las medidas insuficientes para prevenir lesiones y la insatisfacción del paciente, siendo esta última quizás la más importante de estos debido a la implicancia que tendría el resultado estético y funcional del tratamiento en la vida cotidiana del paciente.

Según se desprende de esta revisión, otra etapa de menor frecuencia de demandas es la Fase de Mantención, efectuada una vez finalizada la Protésica mediante controles rutinarios del implante osteointegrado. Dentro de los eventos adversos que se pueden dar en esta etapa, destacan la aparición de enfermedades periimplantarias, cuyo surgimiento tendría relación con condicionantes propias del paciente como lo son enfermedades locales o sistémicas u otro tipo de factores, por lo cual se ha sugerido que algunos de estos factores de riesgo podrían representar contraindicaciones para la realización de tratamiento de implantes dentales (Hwang \& Wang, 2006; Sugerman \& Barber, 2002). Esto último refuerza además la idea planteada previamente de consignar correctamente en el consentimiento informado la historia médica y las condicionantes del paciente que podrían influir en el resultado final del tratamiento. En la Fase de Mantención, la disposición del paciente toma gran relevancia, ya que gran parte del cuidado del implante debe ser realizado por él mismo mediante una correcta higiene para un continuo cuidado de tejidos periimplantarios, por lo que es de suma importancia esta reciprocidad y retroalimentación para el éxito final del tratamiento (Todescan et al., 2012). Se puede concluir que la baja frecuencia de demandas en esta fase se debe a que gran parte de la responsabilidad en este caso es compartida, por lo que el paciente no podría culpar al operador por malpraxis si este no sigue las indicaciones individualizadas que este le entregará para el cuidado correcto del implante.

La alta frecuencia de eventos adversos a nivel transversal del tratamiento, tendría relación con faltas de comunicación no solo entre el clínico y su paciente, sino también entre las distintas disciplinas odontológicas que participan en el equipo multidisciplinario del tratamiento implantológico. Diversos artículos han destacado la importancia de una participación multidisciplinaira en el equipo implantológico en casos particulares, donde este debe ser acompañado de una buena planificación previa y diagnóstico, como así también de los conocimientos adecuados de todas las disciplinas que integran el tratamiento para su realización óptima (Pérez Pérez et al., 2013). La comunicación entre las diferentes especialidades promueve un conocimiento holístico en el equipo, esto desde la suma de contenidos conceptuales, procedimentales e incluso actitudinales en las áreas de anatomía, diagnóstico e imagen, periodoncia, rehabilitación oral, contribuyendo todas en su sinergia al éxito final del tratamiento (Davda \& Davda, 2013). En Chile, los estándares sugeridos por la normativa (Ministerio de Salud, 2013) promueven un alcance multidisciplinario en las competencias del especialista, lo que podría inferirse es adoptado por los diferentes programas en oferta. Sin embargo, la ausencia de reportes de eventos adversos en implantología o de un análisis científico de aquellos casos que han llegado a formalizarse en el ámbito jurídico, podría estar indicando un subdiagnóstico del problema y, por ende, una falta de adopción de medidas no solo preventivas de demandas, sino además de protección para el paciente (Perea-Pérez et al.). 
Para Astudillo et al. (2015), la más afectada de todas las áreas de la odontología es la implantología. Según los autores, la falta de comunicación con los pacientes, así como la gran variedad de procedimientos y modalidades con potencial riesgo asociado a costes económicos, son responsables de colocar a esta especialidad como la principalmente demandada en varios países. En Chile, Portela León estudiando 156 formularios ingresados a la Superintendencia de Salud del área de odontología entre 2005 y 2010, reporta un franco crecimiento en cantidad de estos reclamos con un 10,9\% de los mismos involucrando a la especialidad de implantología, evidenciando diferentes aspectos clínicos y de relación humana como causales. Moscoso Matus \& Smok Vásquez, analizando la casuística de responsabilidad sanitaria odontológica evaluada por el Servicio Médico Legal de Chile (SML) entre 2007 y 2012, indican que solo un $5 \%$ del total de casos involucraron odontólogos, de los cuales un $18 \%$ comprometió a la especialidad de implantología. Contrariamente a lo acontecido en la generalidad de las pericias abordadas por el SML, los autores afirman que en el área odontológica el $35 \%$ de los casos fueron sentenciados por infracción a la Lex Artis. Entre las razones que podrían explicar este fenómeno, y más allá de lo estrictamente técnico, una relación paciente-profesional debilitada o inadecuada, o la escasa formación del profesional en ámbitos odontológico legales, dan cuenta de la posible percepción fallida del especialista a este tipo de adversidad (Moscoso Matus \& Smok Vásquez). Bien vale mencionar que aunque el citado Decreto Exento $\mathrm{N}^{\circ}$ 31 del Ministerio de Salud de Chile (Ministerio de Salud, 2013) menciona entre los requisitos transversales de conocimientos generales para el área odontológica a la Ley 20.584 (Ministerio de Salud, 2012), solo las especialidades de Endodoncia y Ortodoncia incluyen entre sus requisitos de conocimientos teórico-prácticos, a elementos de odontología legal y de responsabilidad profesional. Se ha mencionado que la asimilación y estudio de los eventos adversos, y la construcción de un modelo centrado en la seguridad del paciente, son medidas fundamentales de calidad en atención sanitaria que ya ha dado evidencias de mejorar no solo los resultados de esa atención sino también de disminuir la cantidad de litigios (Rivera Mendoza et al.). Sin embargo, esta propuesta no ha sido llevada de igual modo al ámbito odontológico, probablemente por presentar daños más leves, procedimientos ambulatorios y prácticas fundamentalmente privadas, todos motivos que, en mayor o menor medida, explicarían este posible subdiagnóstico.
La alta frecuencia de eventos adversos en la Fase Quirúrgica denotaría fallas en la capacidad de planificación del implantólogo, como así también la falta de conocimientos anatómicos y de estudio minucioso en el paciente a tratar. Se sugiere profundizar en tópicos inherentes a la prevención, contención y respuesta ante estos eventos adversos en el desarrollo de la especialidad, así como la provisión de herramientas de mediación y asesoramiento jurídico para así lograr prevenir de manera más eficiente los eventos adversos que puedan surgir en el ejercicio de la especialidad de Implantología.

ARROYO-NAVARRETE, M. \& FONSECA, G. M. Malpractice claims at different stages of treatment in oral implantology: A review. Int. J. Odontostomat., 15(2):434-442, 2021.

ABSTRACT: Dental malpractice claims have increased in recent years, and implantology is one of the most litigated specialties. Most of these claims have been characterized by having a multifactorial nature, with errors reported in any of their diagnostic, therapeutic or maintenance phases. The purpose of this review was to establish the stage of implant treatment in which the most malpractice claims were made, establishing a categorization of the errors detected and damages associated with each of the phases that includes rehabilitation treatment using dental implants. A review was carried out with a systematic search of the last 10 years in Pubmed, Scopus, Web of Science and SciELO databases, complemented with a manual search in specialized journals and in Google Academic, of articles from key words in Spanish, English and Portuguese languages. Only 3 articles were identified that met the selection criteria, which affirms the concept of scant attention given to this eventuality. The claims identified in these reports were analyzed on four types of risk in implantology according to the stage of treatment in which they appear. The surgical stage was identified as the one with the highest risk potential of causing adverse events and associated demands. It is suggested to deepen the specific and generic medico-legal aspects of the specialty, as well as in the development of strategies to prevent adverse events and the associated litigation.

KEY WORDS: Oral implantology, malpractice, health risk, prevention.

\section{REFERENCIAS BIBLIOGRÁFICAS}

Astudillo, M.; Chichilla, D. \& Sarabia, L. Mala praxis odontológica: artículo de revisión. Rev. Venez. Invest. Odontol. IADR, 3(2):146$70,2015$.

Bordonaba-Leiva, S.; Gómez-Durán, E. L.; Balibrea, J. M. \& BenetTravé, J. Twenty four years of oral and maxillofacial surgery malpractice claims in Spain: patient safety lessons to learn. Oral Maxillofac. Surg., 23(2):187-92, 2019. 
Camargo, I. B. \& Van Sickels, J. E. Surgical complications after implant placement. Dent. Clin. North. Am., 59(1):57-72, 2015.

Chee, W. \& Jivraj, S. Failures in implant dentistry. Br. Dent. J., 202 (3): $123-9,2007$

Collins Calcaño, J. R.; García Pichardo, S.; Geraldino Chupani, M. A. \& Polanco Aquino, R. T. Colocación de implantes y provisionalización inmediata en el sector estético: manejo quirúrgico y rehabilitador. Reporte de un caso clínico. Rev. Clin. Periodontol. Implantol. Rehabil. Oral,7(2):93-100. 2014.

Corte-Real, A.; Caetano, C.; Dias Pereira, A.; Rocha, S.; Alves, S. \& Nuno-Vieira, D. Risk and limits in dental practice: a portuguese approach to medical-legal evaluation and professional liability. J. Forensic Odontostomatol.,1(38):2-7, 2020.

Davda, L. S. \& Davda, S. V. Implant dentistry: a multidisciplinary approach. J. Interdiscip. Dent., 3(2):52-6, 2013.

De Vico, G.; Ferraris, F.; Arcuri, L.; Guzzo, F. \& Spinelli, D. A novel workflow for computer guided implant surgery matching digital dental casts and CBCT scan. Oral Implantol., 9(1):33-48, 2016.

Esposito, M.; Hirsch, J. M.; Lekholm, U. \& Thomsen, P. Biological factors contributing to failures of osseointegrated oral implants (I). Success criteria and epidemiology. Eur. J. Oral Sci.,106(1):52751,1998

Fonseca, G. M.; Belmar-Durán, M. \& Matamala-Santander, C. Failure to obtain informed consent should also be considered an adverse event. J. Dent. Sci., 15(2):232-3, 2020.

Givol, N.; Taicher, S.; Halamish-Shani, T. \& Chaushu, G. Risk management aspects of implant dentistry. Int. J. Oral Maxillofac. Implants, 17(2):258-62, 2002.

Hapcook Sr., C. P. Dental malpractice claims: percentages and procedures. J. Am. Dent. Assoc., 137(10):1444-5, 2006.

Hashemipour, M. A.; Movahedi Pour, F.; Lotfi, S.; Gandjalikhan Nassab, A. H.; Rahro, M. \& Memaran Dadgar, M. Evaluation of dental malpractice cases in Kerman province (2000-2011). J. Forensic Leg. Med., 20(7):933-8, 2013.

Hwang, D. \& Wang, H. L. Medical contraindications to implant therapy. Part I. Absolute contraindications. Imp. Dent.,15(4): 353-60, 2006.

Isidor, F. Mobility assessment with the Periotest system in relation to histological findings of oral implants. Int. J. Oral Maxillofac. Implants, 13(3):377-83, 1998

Kiani, M. \& Sheikhazadi, A. A five-year survey for dental malpractice claims in Tehran, Iran. J. Forensic Leg. Med., 16(2):76-82, 2009.

Knaak, J. P. \& Parzeller, M. Court decisions on medical malpractice. Int. J. Leg. Med., 128(6):1049-57, 2014.

Kourtis, S. G.; Sotiriadou, S.; Voliotis, S. \& Challas, A. Private practice results of dental implants. Part I: survival and evaluation of risk factors. Part II: surgical and prosthetic complications. Implant Dent., 13(4):373-85, 2004.

Manca, R.; Bruti, V.; Napoletano, S. \& Marinelli, E. A 15 years survey for dental malpractice claims in Rome, Italy. J. Forensic Leg. Med., 58:74-7, 2018

Marinescu Gava, M.; Suomalainen, A.; Vehmas, T. \& Venta, I. Did malpractice claims for failed dental implants decrease after introduction of СВСТ in Finland? Clin. Oral Investig., 23(1):399404, 2018.

Ministerio de Salud. Decreto Exento No 31. Modifica Norma Técnica $N^{\circ} 145$, requisitos específicos de postulación: Descripción de aspectos técnicos y conocimientos mínimos para la certificación de especialidades y subespecialidades médicas y las odontológicas. Santiago de Chile, Gobierno de Chile, 2013.

Ministerio de Salud. Ley 20.584. Regula los Derechos y Deberes que tienen las Personas en Relación con Acciones Vinculadas a su Atención en Salud. Santiago de Chile, Gobierno de Chile, 2012.

Misch, C. E. \& Resnik, R. Mandibular nerve neurosensory impairment after dental implant surgery: management and protocol. Implant Dent., 19(5):378-86, 2010.

Misch, K. \& Wang, H. L. Implant surgery complications: etiology and treatment. Implant Dent., 17(2):159-68, 2008.
Moscoso Matus, K. \& Smok Vásquez, P. Responsabilidad sanitaria en odontólogos. Casuística de casos evaluados en el Servicio Médico Legal de Chile. Rev. Med. Chile,143(3):345-51, 2015.

Perea-Pérez, B.; Santiago-Sáez, A.; Labajo-González, M. E. \& Albarrán-Juan, M. E. Professional liability in oral surgery: legal and medical study of 63 court sentences. Med. Oral Patol. Oral Cir. Bucal, 16(4):526-31, 2011.

Pérez Pérez, O.; Velasco Ortega, E.; Rodríguez, O. \& González Olivares, L. Resultados del tratamiento con implantes dentales osteointegrados en la fase quirúrgica. Rev. Cuba. Estomatol., 50(4):351-63, 2013.

Pinchi, V.; Varvara, G.; Pradella, F.; Focardi, M.; Donati, M. D. \& Norelli, G. Analysis of professional malpractice claims in implant dentistry in Italy from insurance company technical reports, 2006 to 2010 . Int. J. Oral Maxillofac. Implants, 29(5):1177-84, 2014.

Pippi, R.; Spota, A. \& Santoro, M. Medicolegal considerations involving iatrogenic lingual nerve damage. J. Oral Maxillofac. Surg., 76(8):1651.e-1-1651.e13, 2018.

Portela León, F. J. Caracterización de los aspectos bioéticos en los reclamos realizados ante la Superintendencia de Salud en Chile en el área de la Odontología. Acta Bioeth.,19(1):143-52, 2013.

Rees, J. Medicolegal Implications of Dental Implant Therapy. Prim. Dent. J., 2(2):34-8, 2013.

Resnik, R. R. Dental Implants Complications. En: Resnik, R. R. (Ed.). Contemporary Implant Dentistry. 4th ed. Toronto, Elsevier, 2020. pp.771-829.

Rivera-Mendoza, F.; Acevedo-Atala, C.; Perea-Pérez, B.; LabajoGonzález, E. \& Fonseca, G. M. Análisis causa-raíz sobre evento adverso producido en la clínica odontológica docente asistencial, Facultad de Odontología, Universidad de La Frontera, Chile. Int. J. Odontostomat., 11(2):207-16, 2017.

Sugerman, P. B. \& Barber, M. T. Patient selection for endosseous dental implants: oral and systemic considerations. Int. J. Oral Maxillofac. Implants, 17(2):191-201, 2002.

Tatakis, D. N., Chien, H. H. \& Parashis, A. O. Guided implant surgery risks and their prevention. Periodontol. 2000, 81(1):194-208, 2019.

Todescan, S.; Lavigne, S. \& Kelekis-Cholakis, A. Guidance for the maintenance care of dental implants: clinical review. J. Can. Dent. Assoc., 78:c107, 2012

Tolstunov, L. Dental implant success-failure analysis: a concept of implant vulnerability. Implant Dent., 15(4):341-6, 2006

Triana Estrada, J. La ética: Un problema para el odontólogo. Acta Bioeth., 12(1):75-80, 2006.

Van Assche, N.; Vercruyssen, M.; Coucke, W.; Teughels, W.; Jacobs, R. \& Quirynen, M. Accuracy of computer aided implant placement. Clin. Oral Implants Res., 23(Suppl. 6):112-23, 2012.

Villarroel, M. J. Corte confirma condena a dentista: deberá pagar $\$ 82$ millones a paciente tras perforarle un nervio. Radio Bío Bío, 2019. Disponible en: https://www.biobiochile.cl/noticias/nacional/regionmetropolitana/2019/07/04/corte-confirma-condena-a-dentistadebera-pagar-82-millones-a-paciente-tras-perforarle-un-nervio.shtml

World Health Organization (WHO). Quality of care: patient safety. Resolution WHA55.18. Ginebra, World Health Organization, 2002. Disponible en: https://apps.who.int/gb/archive/pdf_files/WHA55/ ewha5518.pdf

Dirección para correspondencia:

Dr. Gabriel M. Fonseca

Centro de Investigación en Odontología Legal y Forense (CIO)

Facultad de Odontología

Universidad de La Frontera

Francisco Salazar 01145

Temuco - CHILE

E-mail: gabriel.fonseca@ufrontera.cl 